\title{
Effekt der oralen Gabe von Kieselerde auf die Haarqualität
}

\author{
The Effect of Oral Applied Silica on Hair Quality
}

Autor

Institut

\section{W. Gehring}

Hautklinik am Klinikum der Stadt Karlsruhe gGmbH

\section{Bibliografie}

DOI http://dx.doi.org/

10.1055/s-0030-1255611

Online-Publikation: 29. 7. 2010

Akt Dermatol 2010; 36:

414-417 @ Georg Thieme

Verlag KG Stuttgart · New York ISSN 0340-2541

Korrespondenzadresse

Prof. Dr. Wolfgang Gehring

Direktor der Hautklinik

am Klinikum der Stadt

Karlsruhe $\mathrm{gGmbH}$

Moltkestr. 120

76133 Karlsruhe

w.m.gehring@t-online.de

\section{Zusammenfassung \\ $\nabla$}

Es wurde der Effekt der oralen Gabe von Kieselerde bei reduzierter Haarqualität mit spröden und brüchigen Haaren untersucht. 79 Probanden wurden für 6 Monate mit $3 \times 350 \mathrm{mg}$ Kieselerde täglich behandelt. Primärer Bewertungsparameter für die Haarqualität war das Quellverhalten

\section{Einleitung \\ $\nabla$}

Kieselerde wird traditionell zur Verbesserung der Haut, der Haare, der Nägel und des Bindegewebes eingesetzt. Kieselerde ist im wesentlichen Siliziumdioxid $\left(\mathrm{SiO}_{2}\right.$, Kieselsäure). Silizium ist ein essentielles Spurenelement und muss mit der Nahrung zugeführt werden. Es kommt im menschlichen Körper am häufigsten in den Wachstumszonen der Knochen, aber auch im Bindegewebe, in der Haut, in den Haaren und in den Nägeln vor [1]. Ein Kieselsäuremangel wirkt sich negativ auf den Organismus aus und kann z. B. durch erhöhte Brüchigkeit von Haaren und Nägeln auffallen, die durch Kieselerde therapierbar ist [2]. Ziel dieser offenen, einarmigen Studie war es, in einer 6 Monate dauernden Untersuchung den Effekt von oral gegebener Kieselerde in Form eines Nahrungsergänzungsmittels auf die Haarqualität zu überprüfen. Spröde, brüchige und splitternde Haare stellen ein sehr häufiges Phänomen dar, das vom Betroffenen mit großem Leidensdruck wahrgenommen wird und den Wunsch nach einer effektiven Behandlung weckt, da mit der reduzierten Haarqualität ein Attraktivitätsverlust verbunden wird. Ursachen für reduzierte Haarqualität sind vor allen Dingen - parallel zur trockenen Haut - eine Sebostase. Hinzu kommen Pflegefehler. Hier sind in erster Linie zu häufiges Waschen der Haare mit austrocknenden Tensiden, mechanische Irritation durch Bürsten, chemische Belastung durch Dauer- des Haares nach Inkubation mit 2,5 m ( $\mathrm{m}=$ molar) $\mathrm{NaOH}$. Sekundäre Parameter waren die Haardicke und die Selbstbeurteilung durch die Probanden. Die Studie hat eine Verbesserung sämtlicher Bewertungsparameter erkennen lassen. Damit wurde die Effektivität von Kieselerde bei der Behandlung einer reduzierten Haarqualität belegt.

welle und Tönen sowie übermäßig häufiges und heißes Föhnen zu nennen.

\section{Material und Methodik}

\section{Hauptparameter - Quellverhalten}

\section{des Haares durch $\mathrm{NaOH}$}

Als Hauptparameter für die Beurteilung der Auswirkung der oral applizierten Kieselerde auf die Haarqualität wurde das Quellverhalten des Haares nach Inkubation mit $\mathrm{NaOH}$ erfasst. Dieses Verfahren geht auf Zaun zurück und hat sich bei klinischen Untersuchungen an Nagelkeratin bewährt [3-5]. Eigene, nicht publizierte Erfahrungen zur Übertragbarkeit der Methode auf Haarkeratin liegen vor. Haare und Nägel bestehen vorwiegend aus Keratin und sind in ihrer chemischen Zusammensetzung sehr ähnlich [6], sodass auch aus biochemischer Sicht ein vergleichbares Verhalten von Haaren und Nägeln verständlich ist. Von einer reduzierten Haarqualität kann ausgegangen werden, wenn das Quellverhalten des Haares nach Inkubation mit 2,5 $\mathrm{m} \mathrm{NaOH}$ kleiner ist als der Faktor 2,5.

Die Inkubation und mikroskopische Vermessung des Haares, das standardisiert $1 \mathrm{~cm}$ oberhalb des Haarbodens abgetrennt worden ist, erfolgte in vitro unter einem locker aufliegenden Deckgläschen. Die Inkubationsdauer mit 2,5 m NaOH betrug 15 Minuten. Wird während der Therapie 
der Quellfaktor von 2,5 erreicht oder überschritten, so kann von einer Verbesserung und Normalisierung der Haarqualität ausgegangen werden.

\section{Nebenparameter - Absolute Haardicke und klinische Selbstbeurteilung der Haarqualität durch den Probanden}

Vor Beginn der Untersuchung sowie nach 3 und nach 6 Monaten Einnahme der Kieselerde-Kapseln wurde die absolute Haardicke mikroskopisch mittels einer Mikrometerskala bei einer Vergrößerung von $10 \times 10$ bestimmt.

Zur Dokumentation der klinischen Selbstbeurteilung durch den Probanden (Global Impression) diente eine stufenlose Skala von 0 - 10, bei der 0 für „sehr schlecht“ und 10 für „sehr gut“ stand. Sämtliche Parameter wurden vor Beginn der Behandlung (T0), nach 3 Monaten (T3) und nach 6 Monaten (T6) erfasst und dokumentiert.

\section{Statistische Auswertung}

Nach vorheriger Varianzanalyse mit dem Friedmann-Test wurde der Wilcoxon-Paardifferenzentest für verbundene Stichproben durchgeführt. Es erfolgte ein Vergleich zur Baseline nach 3 und nach 6 Monaten (T3/T0 und T6/T0) und zwischen dem 3. und 6. Monat. Hauptparameter und die einzelnen Nebenparameter wurden getrennt ausgewertet. In die statistische Auswertung gingen jeweils die Absolutwerte ein: für den Hauptparameter der absolute Quellindex, für die Haardicke die absolute Haardicke in $\mathrm{mm}$ und für die Selbstbeurteilung der Skalenwert. Von Kieselerde ist eine Verbesserung der Haarqualität zu erwarten. Aus diesem Grund wurde die Fragestellung einseitig formuliert. Das vorgegebene Signifikanzniveau war 5\%. Die Darstellung der Ergebnisse in den Abbildungen bezieht sich auf die Mittelwerte.

\section{Probandenkollektiv}

Es wurden 83 Probanden mit splitternden, brüchigen und spröden Haaren eingeschlossen, bei denen von einer reduzierten Haarqualität ausgegangen werden kann, da die durch 2,5 m Natronlauge provozierte Quellung des Haares kleiner als der Faktor 2,5 war. 79 Probanden haben die Studie nach Plan abgeschlossen und sind in die Auswertung der Ergebnisse eingegangen.

\section{Studienpopulation}

Die Studienpopulation setzte sich folgendermaßen zusammen (๑ Tab. 1):

Tab. 1 Studienpopulation.

\begin{tabular}{|llll|}
\hline & gesamt & männlich & weiblich \\
\hline Anzahl der Probanden & 83 & 21 & 62 \\
\hline Durchschnittsalter & 40,6 & 41,5 & 40,3 \\
\hline Altersgrenzen & $18-63$ & $20-63$ & $18-61$ \\
\hline
\end{tabular}

\section{Einschlusskriterien}

- weibliche und männliche gesunde Probanden

- Alter zwischen 18 und 65 Jahren.

- Unterschriebene Einverständniserklärung liegt vor.

- splitternde, brüchige und spröde Haare und Quellfaktor der Haare $\leq 2,5$

\section{Ausschlusskriterien}

- Probanden unter 18 Jahren und über 65 Jahren

- Quellverhalten der Haare durch 2,5 m NaOH > Faktor 2,5

- pathologische Erkrankungen der Haare und der Kopfhaut

- Einnahme/Verwendung von Produkten, von denen ein Effekt auf die Haare zu erwarten ist (siehe unerlaubte Produkte)

- systemische Grunderkrankungen

- schwangere oder stillende Frauen

- mangelnde Compliance

- fehlende Einverständniserklärung

- Der Proband nimmt an einer weiteren Studie teil oder hat innerhalb der letzten 30 Tage an einer Studie teilgenommen.

- bekannte Überempfindlichkeit oder Allergie gegen einen Inhaltsstoff des Prüfprodukts

\section{Unerlaubte Produkte}

Während der Studie ist die zusätzliche Verwendung der folgenden Produkte mit Einfluss auf die Haarqualität nicht erlaubt:

- topische oder systemische Hormonpräparate, die die Haarqualität beeinflussen

- Gelatinepräparate

- Biotinpräparate

\section{Prüfprodukt}

Untersucht wurden Kieselerde-Kapseln als Nahrungsergänzungsmittel für Haare, Haut und Nägel, die von GlaxoSmithKline (GSK) Consumer Healthcare GmbH \& Co. KG zur Verfügung gestellt worden waren.

Eine Kapsel des Prüfpräparates enthielt 350 mg Kieselerde. Weitere Zutaten der Kapsel waren Gelatine, Füllstoff Kalziumphosphat, Trennmittel Kalziumsalze von Speisefettsäuren, Farbstoff und Titandioxid.

Über einen Studienzeitraum von 6 Monaten wurde $3 \times$ tgl. 1 Kapsel Kieselerde mit jeweils 350 mg Wirkstoff verabreicht.

Vor Beginn der Studie wurde die berufsrechtliche Beratung der Ethikkommission der Landesärztekammer Baden-Württemberg eingeholt.

\section{Ergebnisse}

\section{Hauptparameter}

Quellverhalten durch Inkubation mit $\mathrm{NaOH}$

Vor Beginn der Untersuchung lag der absolute Quellindex bei 2,2. Nach drei Monaten und nach 6 Monaten zeigte sich eine Verbesserung des Quellverhaltens mit einem Wert von jeweils 2,5 (৫ Abb. 1).

Die Daten sind wie folgt charakterisiert ( Tab. 2):

Tab. 2 Statistische Werte für Quellverhalten durch $\mathrm{NaOH}$.

\begin{tabular}{|llll} 
& T0 & T3 & T6 \\
\hline Number of values & 79 & 79 & 79 \\
\hline Minimum & 1,0 & 2,0 & 2,0 \\
\hline 25\%-Perzentile & 2,1 & 2,2 & 2,3 \\
\hline Median & 2,2 & 2,4 & 2,4 \\
\hline 75\%-Perzentile & 2,4 & 2,7 & 2,6 \\
\hline Maximum & 2,5 & 4,0 & 3,8 \\
\hline Mean & 2,207 & 2,4804 & 2,487 \\
\hline Std. Deviation & 0,2334 & 0,3740 & 0,3220 \\
\hline Std. Error & 0,02626 & 0,04208 & 0,03623 \\
\hline
\end{tabular}




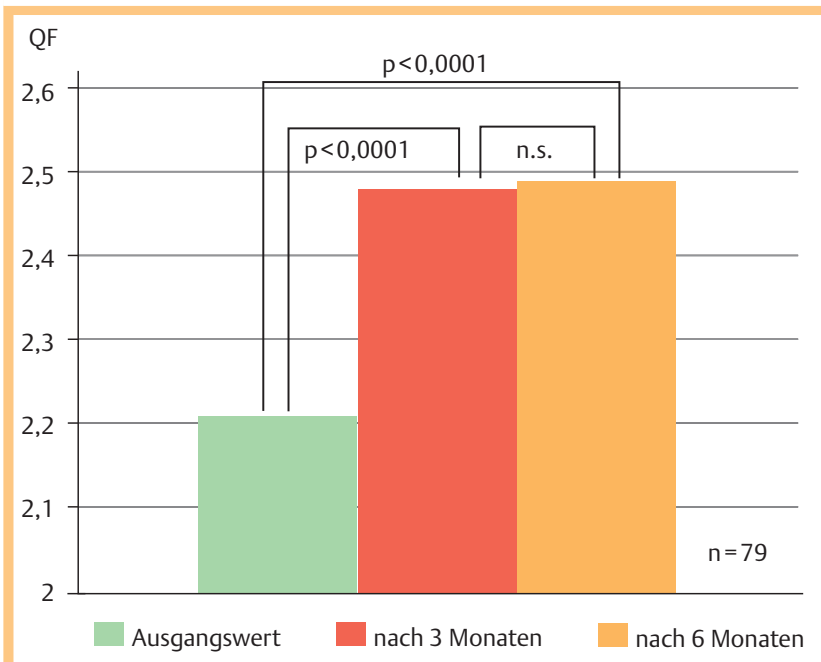

Abb. 1 Verbesserung des Quellverhaltens des Haarkeratins nach Inkubation mit 2,5 m NaOH unter Behandlung mit Kieselerde (QF, Quellfaktor; n. s., nicht signifikant).

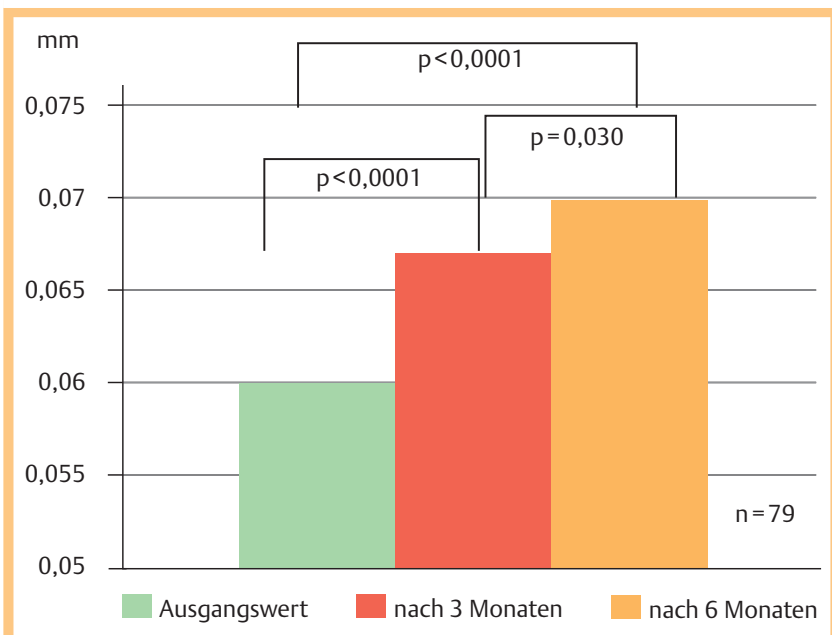

Abb. 2 Zunahme der Haardicke durch Behandlung mit Kieselerde.

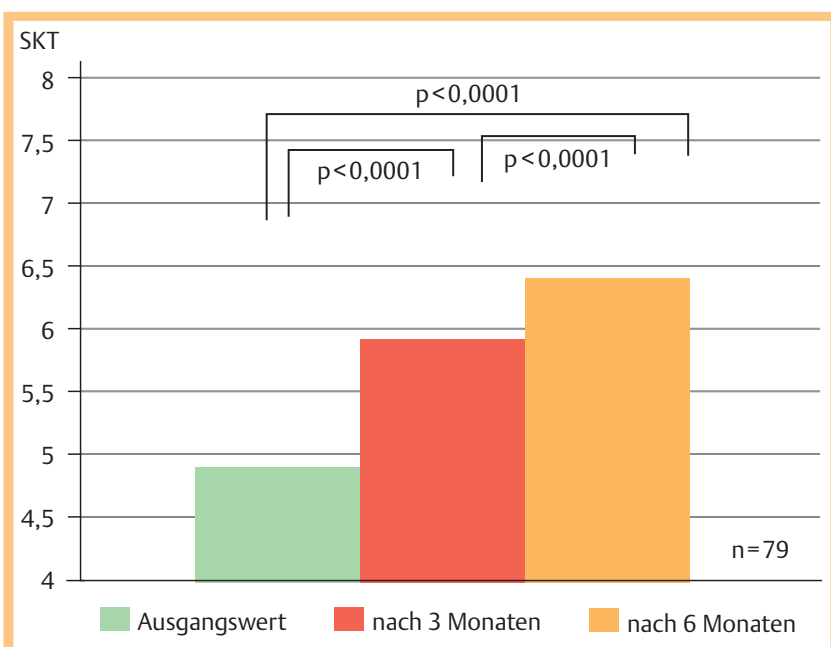

Abb. 3 Selbstbeurteilung der Haarqualität durch die Probanden unter Behandlung mit Kieselerde (SKT, Skalenteile; 0 = sehr schlecht, $10=$ sehr gut).
Es zeigte sich folgende Signifikanzverteilung:

- T3/T0: $\mathrm{p}<0,0001$

T6/T0: $\mathrm{p}<0,0001$

- T6/T3: n.s.

\section{Nebenparameter}

Haardicke

Vor Beginn der Behandlung fand sich im Mittel eine Haardicke von $0,0602 \mathrm{~mm}$. Nach 3 Monaten war eine Verbesserung auf $0,0668 \mathrm{~mm}$ und nach 6 Monaten eine weitere Verbesserung der Haardicke auf 0,0701 mm gegeben ( $\bullet$ Abb. 2).

Die Daten sind wie folgt charakterisiert ( Tab.3):

Tab. 3 Statistische Werte für Haardicke.

\begin{tabular}{|llll|}
\hline & T0 & T3 & T6 \\
\hline Number of values & 79 & 79 & 79 \\
\hline Minimum & 0,03 & 0,04 & 0,05 \\
\hline 25\%-Perzentile & 0,05 & 0,057 & 0,06 \\
\hline Median & 0,06 & 0,07 & 0,07 \\
\hline 75\%-Perzentile & 0,07 & 0,075 & 0,08 \\
\hline Maximum & 0,08 & 0,11 & 0,097 \\
\hline Mean & 0,06026 & 0,0669 & 0,0701 \\
\hline Std. Deviation & 0,0132 & 0,013 & 0,124 \\
\hline Std. Error & 0,00148 & 0,00146 & 0,00139 \\
\hline
\end{tabular}

Folgende Signifikanzverteilung ließ sich errechnen:

- T3/T0: $\mathrm{p}<0,0001$

T6/T0: $\mathrm{p}<0,0001$

- T6/T3: $\mathrm{p}<0,03$

Selbstbewertung durch den Probanden

Vor Beginn der Behandlung wurde die Haarqualität mit einer Skalierung von 4,911 angegeben. Im Verlauf der Studie steigerte sich dies nach 3 Monaten auf 5,854 und nach 6 Monaten auf 6,424 ( Abb. 3).

Die Daten sind wie folgt charakterisiert ( Tab.4):

Tab. 4 Statistische Werte für Selbstbewertung.

\begin{tabular}{|c|c|c|c|}
\hline & TO & T3 & T6 \\
\hline Number of values & 79 & 79 & 79 \\
\hline Minimum & 2,5 & 3,0 & 3,0 \\
\hline $25 \%$-Perzentile & 4,0 & 5,0 & 5,0 \\
\hline Median & 5,0 & 6,0 & 6,0 \\
\hline $75 \%$-Perzentile & 6,0 & 7,0 & 7,75 \\
\hline Maximum & 9,0 & 9,5 & 10,0 \\
\hline Mean & 4,911 & 5,854 & 6,424 \\
\hline Std. Deviation & 1,260 & 1,371 & 1,526 \\
\hline Std. Error & 0,1418 & 0,1542 & 0,1716 \\
\hline
\end{tabular}

Folgende Signifikanzverteilung war gegeben:

- T3/T0: $\mathrm{p}<0,0001$

- T6/T0: $\mathrm{p}<0,0001$

$\rightarrow$ T6/T3: $\mathrm{p}<0,0001$ 


\section{Beurteilung der Verträglichkeit}

Ein Proband hat wegen stärkerer Magenbeschwerden die Studie abgebrochen. Zwei Probanden fanden die Verträglichkeit ohne Mahlzeiten „schlecht“. Allerdings wurde von ihnen die Studie nicht abgebrochen. Unabhängig von Unverträglichkeiten haben 2 Probanden die Studie vorzeitig beendet. 13 Probanden haben die Verträglichkeit als „sehr gut“ bezeichnet. 64 Probanden fanden die Verträglichkeit „gut“. Mehrere Probanden haben glaubhaft über ein verstärktes Haarwachstum berichtet.

Insgesamt kam es zu 4 Drop outs:

- 41 Jahre, weiblich: Magenunverträglichkeit

- 55 Jahre, weiblich: keine Nennung von Gründen, allerdings keine Unverträglichkeiten

- 30 Jahre, weiblich: während Studie Schwangerschaft eingetreten

- 48 Jahre, weiblich: Ausschluss als Studienassistentin

\section{Diskussion}

\section{$\nabla$}

Das Prüfpräparat hat sich anhand sämtlicher Parameter als effektiv erwiesen. Beim Quellverhalten des Haares nach Inkubation mit $\mathrm{NaOH}$ wurde bereits nach drei Monaten Behandlung der Normwert von 2,5 erreicht. Es besteht eine enge Korrelation zur klinischen Selbstbeurteilung durch die Probanden. Anhand der Selbstbeurteilung ließ sich nach 3 und 6 Monaten eine statistisch signifikante Verbesserung der Haarqualität gegenüber dem Ausgangswert belegen. Diese Korrelation belegt die Eignung des Quellverhaltens des Haarkeratins durch $\mathrm{NaOH}$ als validen Bewertungsparameter zur Beurteilung der Haarqualität. Ursprünglich geht dieses Verfahren auf Zaun zurück, der es zur Beurteilung der Nagelqualität eingesetzt hat [7]. Wir haben mit diesem Parameter den Effekt von Gelatine [4], Biotin [5] und in einer nicht publizierten Studie von Kieselerde am Nagel bemessen und konnten wiederholt auf eine enge Korrelation dieses Parameters zur klinischen Besserung hinweisen. Die vorliegende Studie belegt in klarer Form die Eignung und Übertragbarkeit dieses Parameters auf das Haarkeratin. Neben der Verbesserung des Quellverhaltens nach Inkubation mit $\mathrm{NaOH}$ und der korrelierenden Selbstbeurteilung ist die objektive Verbesserung der Haardicke durch Kieselerde bemerkenswert. Zu Beginn fand sich im Mittel eine Haardicke von 0,0602 mm. Nach 3 Monaten betrug die Haardicke 0,0668 mm und nach 6 Monaten sogar 0,0701 mm. In der Literatur gibt es wiederholte Hinweise auf die Bedeutung von Kieselerde für die Funktion der Haut sowie die Struktur von Haaren und Nägeln $[2,8]$. Barel et al. haben eine Reduktion der Brüchigkeit von Nägeln und Haaren nach Lichtschädigung nachgewiesen [9]. Im Rahmen einer randomisierten Doppelblindstudie konnte durch Siliziumsupplementierung eine Verbesserung der Haarstruktur und Haarfestigkeit belegt werden [10]. Somit reiht sich die vorliegende Untersuchung am Beispiel der Verbesserung der Haarqualität in die Beobachtungen zum Nutzeffekt der oralen Gabe von Kieselerde ein.

Der Mechanismus der positiven Wirkung von Kieselerde auf Haare und Nägel ist nicht gänzlich geklärt, kann aber vermutlich auf den erhöhten Gehalt von Hydroxyprolin in der Dermis zurückgeführt werden [11]. Silizium bewirkt einen Anstieg des Hydroxyprolins, das einen wichtigen Bestandteil des Kollagens darstellt [12]. Die Aktivität der Prolylhydroxylase arbeitet siliziumabhängig und gilt als Indikator für die Geschwindigkeit der Kollagenbiosynthese [13].
Die orale Gabe von Kieselerde kann aufgrund der vorliegenden Untersuchung zur Verbesserung der Haarqualität empfohlen werden. Der Nutzeneffekt ist aufgrund der engen Korrelation aller drei Bewertungsparameter unstrittig belegt. Betont werden muss ebenfalls die gute bis sehr gute Verträglichkeit des Prüfpräparates.

\section{Danksagung \\ $\nabla$}

Die Studie und die Publikation wurden von GlaxoSmithKline (GSK) Consumer Healthcare GmbH \& Co. KG unterstützt.

\section{Abstract}

\section{The Effect of Oral Applied Silica on Hair Quality}

The effect of oral applied silica on reduced hair quality with brittle and splitting hairs was investigated. Over a treatment period of 6 month $3 \times 350 \mathrm{mg}$ silica daily were applied on 79 volunteers. The primary parameter for the hair quality was the swelling behaviour of the hair after incubation with $2.5 \mathrm{~m} \mathrm{NaOH}$. Secondary parameters were the thickness of the hair and the clinically evaluation by the volunteers. The study could demonstrate an improvement of all parameters. The efficiency of systemically applied silica against reduced hair quality is proved.

\section{Literatur}

1 Schwarz K. Significance and functions of silicon in warm-blooded animals. Review and outlook. In: Bendz G, Lindquist I (eds). Biochemistry of Silicon and Related Problems. New York: Plenum Press, 1978: 207230

2 Lassus A. Colloidal silicic acid for oral and topical treatment of aged skin, fragile hair and brittle nails in females. J Int Med Res 1993; 21: 209-215

3 Zaun H. Der Nagel-Quellfaktor als Kriterium für Wirksamkeit und aussichtsreichen Einsatz von Nageltherapeutika bei brüchigen und splitternden Nägeln. Ärztl Kosm 1981; 11: 242 - 244

4 Gehring W, Gloor $M$. Verbesserung der Nagelqualität durch Gelatine. Akt Dermatol 1992; 18: 364-366

5 Gehring W. Der Einfluss von Biotin bei reduzierter Nagelqualität. Akt Dermatol 1996; 22: 20-25

6 Stüttgen G, Schaefer H. Die Nägel. In: Stüttgen G, Schaefer H (eds). Funktionelle Dermatologie. Berlin, Heidelberg, Tokyo: Springer, 1994: 99 101

7 Zaun H, Becker H. Die Quelleigenschaften von Nagelmaterial in Natronlauge bei der Bestimmung mit einer standardisierten Methode. Ärztl Kosm 1976; 6: 115-119

8 Lassus A. Colloidal silicic acid for the treatment of psoriatic skin lesions, arthropathy and onychopathie. A pilot study. J Int Med Res 1997; 25: 206-209

9 Baral A, Calomme M, Timchenko A et al. Effect of oral intake of cholinestabilized orthosilicic acid on skin, nails and hair in women with photodamaged skin. Arch Dermatol Res 2005; 297: 147-153

10 Wickett RR, Kossmann E, Baral A et al. Effect of oral intake of cholinestabilized orthosilicic on hair tensile strength and morphology in women with fine hair. Arch Dermatol Res 2007; 299: 499-505

11 Scheinfeld N, Dahdah MJ, Scher R. Vitamins and minerals: their role in nail health and disease. J Drugs Dermatol 2007; 6: 782 - 787

12 Carlisle EM. Silicon. In: Frieden E (ed). Biochemistry of the Essential Ultratrace Elements. New York: Plenum Press, 1984: 257-291

13 Reffitt DM, Ogston N, Jugdaohsingh $R$ et al. Orthosilicic acid stimulates collagen type I synthesis and osteoblastic differentiation in human osteoblast-like cells in vitro. Bone 2003; 32: 127-135 